\title{
Current Treatment Options for Patients with Myotonic Dystrophy Type 2
}

\section{Federica Montagnese ${ }^{1, *} \odot$}

\author{
Address \\ *,1Department of Neurology, Friedrich-Baur-Institute, Ludwig-Maximilians-University, \\ Munich, Germany \\ Email: Federica.montagnese@med.uni-muenchen.de \\ Published online: 27 September 2021 \\ (๑) The Author(s) 2021
}

This article is part of the Topical Collection on Neuromuscular Disorders

Keywords Myotonic dystrophy type 2 - PROMM - DM2 - Therapy - Care - Management

\section{Abstract}

Purpose of the review Myotonic dystrophy types 1 and 2 are frequent forms of muscular dystrophies in adulthood. Their clinical differences need to be taken into account for the most appropriate treatment of patients. The aim of this article is to provide an overview on the current and upcoming therapeutic options for patients with myotonic dystrophy type 2 (DM2).

Recent findings At the moment, no disease-modifying therapies are available for DM2; next-generation therapies may however be available in the near future. In the meanwhile, the symptomatic management of patients has greatly improved, thank to the production of consensus-based standards of care and the growing evidence of efficacy of anti-myotonic drugs, promising employment of cannabinoids for symptom's relief, regular monitoring, and early detection of treatable extra-muscular manifestations.

Summary The treatment of DM2 is currently symptomatic and relies on the coordinated intervention of a multidisciplinary team. It remains to be determined whether upcoming causal therapies for myotonic dystrophy type 1 will be applicable also in DM2. 


\section{Introduction}

Myotonic dystrophy type 2 (DM2, \#602,668) is a rare, autosomal dominant, multi-systemic disease caused by a $\operatorname{CCTG}^{(>75)}$ repeat expansion mutation in the intron 1 of the cellular nucleic acid binding protein gene (CNBP 3q21.3, previously known as ZFN9) [1]. DM2 represents, together with the myotonic dystrophy type 1 (DM1), a frequent form of muscular dystrophy; its prevalence worldwide is highly heterogeneous. The highest prevalence is observed in central and northern Europe (especially Germany, Poland, Finland, Czech Republic), where it is as frequent as DM1, whereas it is far less prevalent in the rest of the world and virtually absent in Asia [2, 3].

Despite some general clinical similarities as combination of weakness and myotonia, multisystemic involvement, and neurophysiological and histological features, striking clinical differences help discriminate DM2 from DM1. DM2 lacks a congenital form and the first clinical manifestations usually begin after the 3rd decade of life with muscular symptoms as weakness, musculoskeletal pain, stiffness, myotonia, fatigue, and exercise intolerance which usually represent the reason for the first referral to neurologists. At onset, the neurological examination may be normal or reveal a mild axial and proximal muscle weakness; the $\mathrm{CK}$ is usually mildly elevated and EMG, when performed, not always detects classical myotonic runs, that may sometimes disguise as other forms of pathological spontaneous activity (mostly positive sharp waves) $[2,4]$. The initially milder, unspecific phenotype of myalgia and weakness, can therefore long go overlooked, with reported average diagnostic delays of 7-14 years $[5,6]$. Extra-muscular manifestations as early-onset cataracts, insulin resistance/diabetes, thyroid dysfunction, cardiac arrhythmias, or cardiomyopathies may precede the onset of muscular complaints or occur during disease progression. Differently from DM1, a severe cognitive impairment is not present in DM2. The apparently milder phenotype of DM2 in comparison to the majority of DM1 patients is however not perceived as such by patients. Studies evaluating patients' reported outcomes in DM2 have documented a high disease burden impacting patients' physical, psychological, and social functioning to an extent similar to that observed in DM1 patients. In particular, pain, inability to perform activities, and fatigue were reported as the symptoms that most impair patients' life $[7,8]$. For these reasons, it is important to adopt the most appropriate interventions in order to improve patients' quality of life. Due to the current lack of causal therapies, in this review, I will focus on the currently suggested symptomatic treatment of DM2 manifestations. After a brief overview of the most relevant molecular mechanisms involved in DM2 pathogenesis, I will review the current treatment options for the most typical muscular complaints. In the second part of the review, I will provide a synopsis of the suggested standards of care for the management of extra-muscular manifestations, referring to recently published consensus-care recommendations for further details on these aspects $[9 \bullet \bullet, 10 \bullet \bullet, 11 \bullet \bullet]$. In the last section, I will then briefly discuss the most promising molecular approaches as potential disease-modifying therapies under investigation in DM1, considering in particular those that might find application also in DM2.

\section{Pathogenesis}

The complex and heterogeneous clinical manifestations of DM2, with their similarities and differences from DM1, are the result of even more complex and still partly unknown pathogenic mechanisms that can be classified into three main processes: (i) haploinsufficiency of $C N B P$, (ii) RNA toxic gain of function, and (iii) RAN translation with production of toxic gain of function proteins.

The precise function of $C N B P$ is still quite elusive; it is believed to act as a transcriptional and post-transcriptional gene regulator by relieving secondary 
structures on target mRNAs that exhibit G-rich sequence stretches [12]. Studies on CNBP expression in DM2 retrieved conflicting results; initially, CNBP mRNA and protein expression seemed not affected by gene haploinsufficiency, whereas more recent studies showed mutant pre-mRNA transcripts with resulting deficiency of mRNA and protein levels associated with a reduced translation rate of CNBP target mRNAs [13]. The contribution of CNBP haploinsufficiency to the phenotype of DM2 has been demonstrated in a $\mathrm{Znf9}^{+/-}$mouse model, that replicated some of the clinical features of DM2, namely, muscle wasting and histological features, presence of myotonic discharges, cataract, and cardiac involvement [14].

The additional pathogenic mechanism of RNA toxic gain of function is the best studied thus far and the one with wide expert's agreement. Expanded, untranslated RNA CCUG repeats form hairpin structures, accumulate in the nuclei, and alter the physiological RNA processing mechanisms of transcription, RNA stability, splicing, and translation. These hairpin-structured mutant RNAs show high affinity for some RNA-binding proteins, especially CUGBP1/ CELF1, MBNL1 (Muscleblind-like splicing regulator 1), and rbFOX1. CUGBP1 shuttles between the nucleus and the cytoplasm and regulates several aspects of RNA processing; elevated levels of nuclear CUGBP1 pool and GSK3b are found in skeletal muscle and heart of DM1 promoting misregulation of alternative splicing with histopathological changes and muscle wasting $\left[15^{\bullet}, 16\right.$, $17 \bullet$. While CELF1/GSK3b pathway is definitely overexpressed in DM1, this has not been unanimously proven in DM2, so that the CUGBP1-dependent pathogenic mechanisms may not apply for DM2 [18]. More relevant for DM2 seem the competition of MBNL1 and rbFOX1, likely responsible for the clinical differences between DM1 and DM2 [19••]. Sellier et al. demonstrated that RbFOX1, highly expressed in skeletal muscle, binds with high affinity expanded CCUG repeats and colocalize with nuclear RNA foci in DM2 muscle cells, whereas it does not bind expanded CUG repeats. Given the high affinity of MBNL1 for CCUG repeat expansion as well, rbFOX1 and MBNL1 compete for the CCUG binding sites in a mutually exclusive fashion. Furthermore, increased levels of rbFOX1 release MBNL1 from the nuclear foci; the result is a reduced amount of sequestered MBNL1 into nuclear foci and a reduced MBNL1-dependent spliceopathy, which may explain the less severe phenotype of DM2 in comparison to DM1 [19••].

The most recently identified pathogenic mechanism is the non-canonical (non-ATG) translation of untranslated RNAs (Repeat Associated Non-ATG translation; RAN-translation) with production of toxin proteins, involved in many other repeat expansion diseases as DM1, SCA8, C9Orf72, and fragile$\mathrm{X}$ tremor ataxia syndrome (FXTAS) $[20,21 \bullet \bullet]$. This non-canonical form of translation, not initiated by an ATG codon, occurs in all reading frames in coding and non-coding regions of sense and antisense transcripts carrying repeat expansion mutations [21••]. In DM2, the sense (CCUG) and antisense (CAGG) expanded transcripts are translated into polymeric tetrapeptides (LPAC and QAGR) with aggregating and cytotoxic properties, thus far detected in white and grey matter of DM2 patients [20, 22].

The combination of these pathogenic mechanisms produces a severe derangement in RNA processing at multiple levels in nearly all body systems 
with resulting heterogeneous and complex phenotype characterized by the combination of muscular and extra-muscular involvement.

\section{Treatment of muscular complaints}

\section{Myotonia}

The term myotonia refers to a delayed muscle relaxation after prolonged voluntary contraction, percussion, or electrical stimulation. From a pathophysiological point of view, it results from an increased muscle excitation caused, in DMs, by a reduced function of the chloride channel (ClC1). The mechanisms of ClC-1 deficiency are related to splicing errors and RNA translational defects, mediated by functional depletion of MBNL proteins family and, in DM2, haploinsufficiency of ZNF9/CNBP [14, 23]. The defective ClC-1 is not able to counterpart the $\mathrm{K}$ build-up in the transverse tubules resulting in an increased steady depolarization and resting membrane potential $[24 \bullet, 25]$. It has been furthermore postulated that a defective function of the sodium pump and activated persistent inward sodium currents might also be involved $[26,27]$. The mechanism of percussion-induced myotonia is likely mediated by the activation of the mechanosensitive ion channels TRPV4 [28••]. In fact, the genetic deletion of TRPV4 in an ex-vivo system produced a suppression of

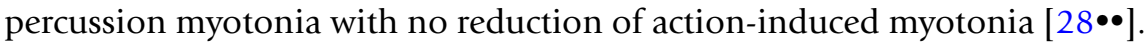

The presence of myotonia can be assessed qualitatively during neurological examination (handgrip and percussion myotonia, climbing stairs, electromyography), semi-quantitatively with patient reported questionnaires (myotonia behaviour scale-MBS) or quantitatively using timed tests (timed up and go, 14 steps stairs), or more elaborated measurements as sensor engineered glove or computerized handgrip myometry [29, 30]. More recently, promising ultrasound techniques as B-mode and shear wave elastography proved effective in documenting the delayed muscle relaxation time, especially in DM1 patients $[31 \bullet, 32 \bullet]$.

In DM2, the assessment of myotonia with the abovementioned methods is complicated by the milder manifestation and different distribution than DM1. Clinically, myotonia manifests in DM2 patients as a mild grip myotonia and/or thighs stiffness with low impact on patient's life [5, 33]. Percussion of the thenar muscles or forearm extensor might reveal myotonia in some

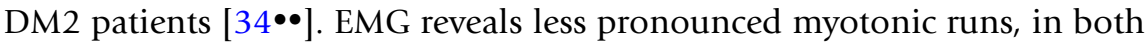
proximal and distal muscles of lower limbs, often without the classical waxing and waning pattern [4].

Even if the severity of myotonia is mostly mild in DM2, this can be enhanced by the cooccurrence of mutations in CLCN1 and SCN4A observed in some DM2 patients or by concomitant endocrinological imbalances as hypothyroidism or pregnancy [35-37]. In cases where the myotonia is impairing patient's activities, the use of an anti-myotonic medication can be considered. Since no drugs with the ability to increase the ClC-1 function are available, the majority of anti-myotonic medications are natrium channel blockers as mexiletine, lamotrigine, carbamazepine, oxcarbazepine, flecainide, 
propaphenone, phenytoin, and ranolazine. Other sodium channel blockers that showed good anti-myotonic effects, thus far only in mouse models, are riluzole, lubeluzole, and safinamide $[38,39 \bullet \bullet]$. Another interesting approach is the activation of the $\mathrm{K}$ channels in order to hyperpolarize the resting membrane potential. Within this category, retigabine, an anti-epileptic drug, proved effective in reducing myotonia by activating the Kv7 channels and thus preventing the after-depolarization from triggering firing of myotonic action potentials. The low tolerability and limited use of retigabine caused its market withdrawal, but the proof of concept that the activation of $\mathrm{K}$ channels reduces myotonia might open the road for new drugs. Lastly, some case reports showed potential efficacy of cannabinoids in the treatment of myotonia and myalgia in DM1 and DM2 patients. Randomized placebocontrolled studies are however required to reliably prove these preliminary results $[40 \bullet 41 \bullet, 42 \bullet]$.

All the above-mentioned anti-myotonic medications are used off-label in myotonic dystrophies. Mexiletine was however recently approved for the treatment of myotonia in non-dystrophic myotonias. Several clinical trials have proved its efficacy in comparison to placebo also in DM1 with good tolerability $\left[43,44 \bullet \bullet, 45^{\bullet}\right]$. The most commonly observed side effects are gastrointestinal symptoms. In order to avoid the risk of cardiac arrhythmias, a cardiological examination must be performed at baseline and 1 week after therapy start.

To monitor the efficacy of anti-myotonic medications in DM2, patients should be advised to use a diary; simple outcome measures as the MBS, the hand opening time and the 14-step stair test should be completed on site $[46 \bullet]$.

Chronic musculoskeletal pain is defined as a "pain perceived in musculoskeletal tissues that lasts or recurs for more than three months, and is characterized by significant functional disability and emotional stress" [47••]. It represents one of the most prevalent symptoms in patients with DM2 [2, $5,48,49]$. Several studies have investigated the characteristics of myalgia in DM2 patients [48-51]. The muscle pain is mostly widespread and fluctuating with a great inter-individual variability; it is usually localized at lower limbs (thighs muscles), low back, neck muscles, and upper limbs/shoulders. There seem to be a broad correspondence between the most affected muscles and the most painful ones. Exercise, cold, and palpation are the most frequently reported factors exacerbating pain in DM2 patients [48, 49]. Common analgesics seldom obtain a satisfactory pain relief, and pain is considered one of the main causes of impaired activities and low life's quality in DM2 [7, 33].

Despite the frequency and entity of the problem, little is still known about the pathophysiology of musculoskeletal pain in DM2. What causes chronic muscle pain in DM2? Why do some patients display a prominent myalgic syndrome and other barely experience pain? Are these mechanisms common to other hereditary myopathies with myalgia? These and many others are still 
open questions and represent an active field of research. Referring to the classical pathophysiologic classification of pain, divided into nociceptive, neuropathic, and, recently, nociplastic, it is still not clear into which category the myalgia of DM2 best fits. Muscular damage might be the trigger of pain (nociceptive), prolonged tissue injury might activate inflammation with peripheral sensitization of high-threshold nociceptors and sensory afferent fibres (neuropathic), and the imbalance between peripheral facilitation and descending inhibition might be involved in central sensitization and pain chronification [52]. Quantitative sensory testing (QST) studies in DM2 patients have documented the presence of mechanical hyperalgesia as expression of peripheral sensitization; furthermore, Moshourab et al. also found increased wind-up ratio and subjective rating of pinprick stimuli in patients with myalgia, which suggests central sensitization [50,51]. Specific molecular changes have been observed in muscles of DM2 patients, where a distinct transcriptomic profile could discriminate myalgia from non-myalgia patients [51].

Psychological factors are then an important contributor to pain exacerbation and disability. Sleep quality, depression, and fatigue are well-known interrelated aspects aggravating pain perception and are frequently observed in patients with DM2 and need to be properly addressed for the best therapeutic management of pain $[53,54]$.

Multimodal analgesia including a combination of pain medications and non-pharmacological treatments should therefore be offered to DM2 patients. The pharmacological approach follows the world health organization (WHO) 4 steps ladder, where the first step corresponds to the use of non-steroidal anti-inflammatory drugs (NSAIDs) with the addition, if needed, of an adjuvant therapy as anticonvulsants (pregabalin, gabapentin), antidepressants (duloxetine, amitriptyline, nortriptyline), muscle relaxants (baclofen, tizanidine), or topical agents (lidocaine or capsaicine patches). The use of opioids should be limited as much as possible due to the high risk of addiction and side effects, starting with the weakest opioids and considering the strongest ones only for short periods. Recent metanalysis showed a moderate opioidsparing effect and pain relief in cannabinoids users with chronic muscle pain [55]. Previously published surveys in myotonic dystrophy cohorts showed that 14 to $33 \%$ of patients have used cannabinoids with subjective improvement of myotonia and myalgia $[40 \bullet 41 \bullet, 42 \bullet$. Thus far, only one case report of two DM2 patients described clear improvement of myalgia in one case, but further well-designed clinical trials are required to evaluate the therapeutic role of cannabinoids in DMs [40•].

A plethora of non-pharmacological interventions can be considered to increase the efficacy of pain medications and include physical treatments (heat and cold applications, massages, stretching), acupuncture, exercise, psychological interventions (e.g., cognitive behavioral therapy), and pain neu-

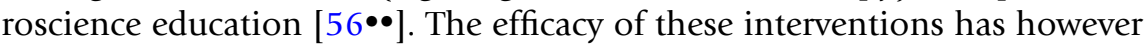
not been specifically investigated in DM2. 


\section{Weakness}

The characteristic distribution of muscular weakness and wasting in DM2 is probably the most striking difference from DM1. DM2 patients present almost invariably an early involvement of the neck flexor muscles; with disease progression, hip flexors and knee extensors are commonly involved; in some cases, a mild weakness of the proximal upper limb muscles may be observed; distal muscles are usually spared $[2,5]$. A mild dysphagia due to a difficult pharyngeal transport is commonly reported by patients, without however significant impairment of food intake nor causing weight loss, need for modified eating habits, or aspiration pneumonia in DM2 [57]. With ageing the muscle weakness and functional impairment gradually increase and some patients will require walking aids as cane, walker, or seldom, wheelchair [5].

The therapeutic options for the symptomatic treatment of weakness are unfortunately very limited. Creatine monohydrate did not show any relevant efficacy in a small cohort of DM2 patients [58]. Several exercise training programs have been tested in myotonic dystrophies, mostly DM1, showing variable results from no relevant changes to improvement of functional motor outcomes and strength measurements, so that at the moment it is not defined what is the best physical exercise protocol to be offered to patients [59•]. Combined resistance and aerobic exercise training recently tested in DM2, improved functional capacity, lean body mass, and blood pressure in DM2 patients $[60 \bullet]$.

New and promising results are expected from a multicentric phase III trial with metformin in DM1. The previous phase II study with orally administered metformin showed a significant improvement of mobility in DM1 patients expressed by increased distance walked on 6-min walk test (6MWT) and improvement of specific gait analysis parameters [ $61 \bullet \bullet]$. The hypothesized mechanisms responsible for these positive results might be pleiotropic, encompassing improved insulin sensitivity, correction of alternative splicing via AMPK-dependent and independent mechanisms and improved metabolic and mitochondrial dysfunction $[62,63 \bullet, 64 \bullet]$. Considering that the abovementioned pathogenic pathways are involved also in DM2, metformin application in DM2 will have to be considered and investigated after conclusion of the phase III trial in DM1.

\section{Monitoring and treatment of extra-muscular involvement}

Some important differences exist between DM1 and DM2 also in regard to the extra-muscular involvement. The frequency and severity of cardiac diseases is higher in DM1 than in DM2, respiratory insufficiency requiring non-invasive ventilation and recurrent respiratory infections are often observed in DM1 patients but are unusual in DM2, the cognitive deficits in DM1 patients may interfere with education and occupation, whereas these are usually mild and unnoticed in DM2 patients. As a result of the aforementioned cardiac and respiratory involvement, the life expectancy of more severely affected DM1 
patients is reduced, due to sudden cardiac death or respiratory complications [65]; more recently, cancer has been added as the third cause of death in patients with DM1 [66]. No specific studies have investigated the life expectancy of DM2 patients in comparison to the general population.

These and other differences between DM1 and DM2 need to be taken into account during patient's monitoring and care, in order to avoid unnecessary health costs and psychological stress to patients. In the last 3 years, consensusbased care recommendations have been produced with the purpose of defining high standards of care for patients with DM1 and DM2 $[9 \bullet \bullet, 10 \bullet \bullet, 11 \bullet \bullet$, 67]. An overview of the monitoring protocols and therapeutic options for the extra-muscular manifestations of DM2 will be here briefly discussed and represented in Fig. 1.

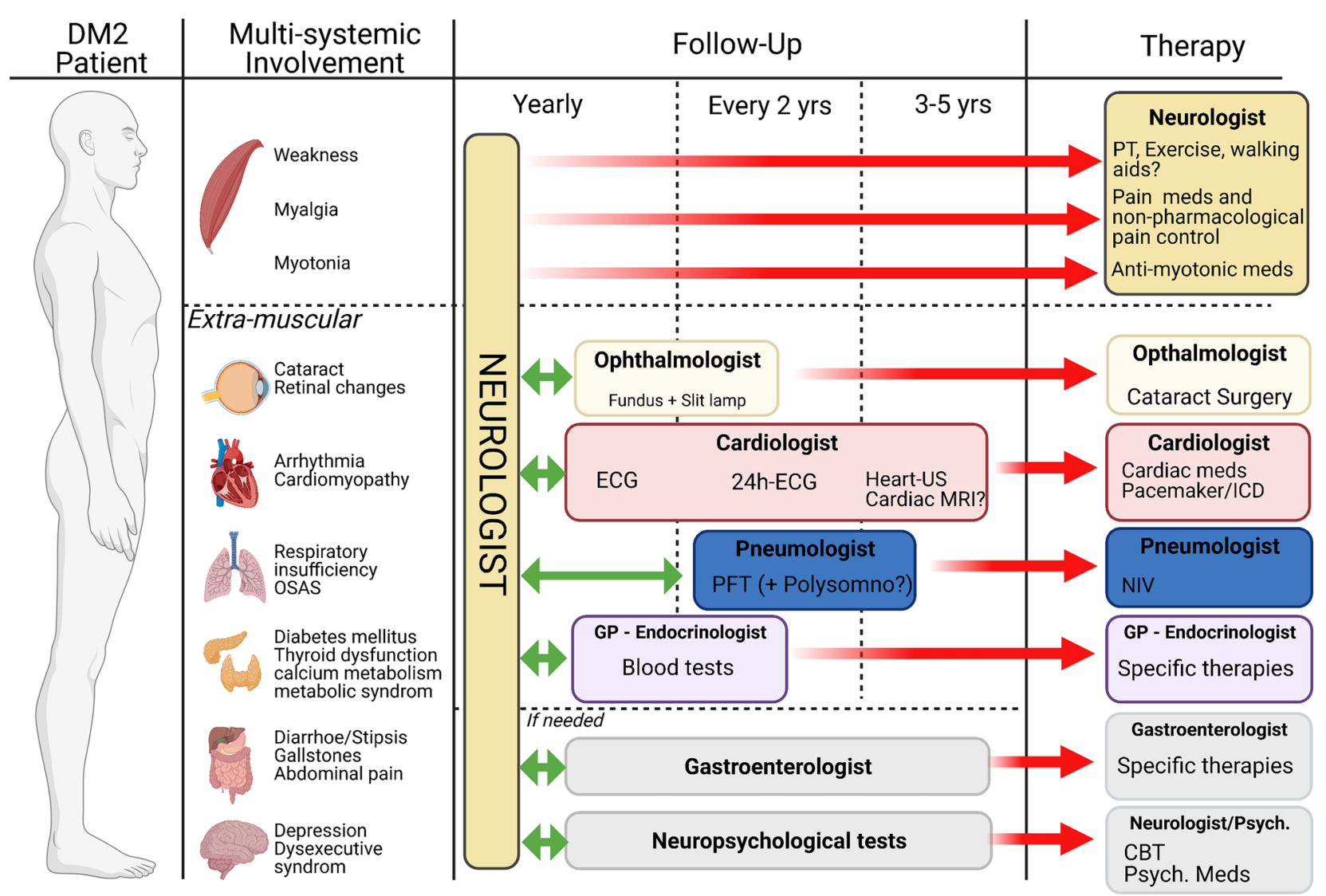

Fig. 1 Suggested monitoring and treatment of DM2 patients. The neurologist is in charge of DM2 patients' care and coordinates the involvement of other specialists that then perform the disease- and organ-specific examinations and treatments. Figure created with Biorender.com. 
Possible manifestations of cardiac involvement in DM2 patients are mostly related to defects of the conduction system (atrioventricular block, atrial, or ventricular tachycardia up to sudden cardiac death) but also cardiomyopathy $\left[68^{\bullet \bullet}\right.$. Males seem to be at higher risk for cardiac complications in comparison to women $\left[69^{\bullet}\right]$.

Therefore, at diagnosis, all DM2 patients should undergo a thorough cardiologic evaluation including 12-lead ECG (if needed completed by HolterECG) and cardiac imaging (echocardiography). If no relevant abnormalities (prolonged PR and QRS intervals, arrhythmias, bradychardia) are detected, these examinations should be repeated every 1-2 years, taking into account the age, the comorbidities, and comedications (e.g., mexiletine!) of the patient $[11 \bullet \bullet$. The detection of pathologically prolonged PR and/or QRS intervals might warrant further investigations as invasive electrophysiological studies, in order to better assess the need of a pacemaker or implantable cardioverter device (ICD) [70•].

DM2 patients often have a high cardiovascular risk due to the presence of diabetes, dyslipidaemia, and metabolic syndrome [71]. In this regard, it is still debated whether patients with DM2 are at higher risk for statin-related adverse reactions. Current recommendations advise, if indicated, the use of statins with strict monitoring of CK and muscular symptoms[11 ••]. Observational studies aimed at the quantification of the risk for statin-related adverse events in patients with myotonic dystrophies would be useful to identify the safest lipid lowering strategy in this population. Today, new lipid lowering drugs with less muscular complications are available (bempedoic acid and PCSK9-inhibitors) and might represent a valuable alternative to statins for DM2 patients.

A restrictive ventilatory dysfunction may be observed in some DM2 patients, usually later in the course of the disease [5]. As in DM1, the causes of respiratory impairment are peripheral (weakness of respiratory muscles) and central (respiratory drive) [72•]. Clinically, patients may experience the classical symptoms of nocturnal hypercapnia (morning headache, excessive daytime sleepiness, reduced concentration), excessive fatigue, dyspnea, and obstructive sleep apnea. The prevalence and progression of respiratory impairment in DM2 are however poorly studied, longitudinal studies are lacking, and direct comparisons between controls, DM1, and DM2 patients were performed on very few DM2 patients, documenting a less severe respiratory involvement in DM2 in comparison to DM1, with only $6-15 \%$ of patients requiring noninvasive ventilation (NIV) [73, $74 \bullet \bullet$. In asymptomatic patients, pulmonary function tests (PFTs) should be performed at diagnosis and every 2 years; if abnormalities are detected or respiratory symptoms observed, the same

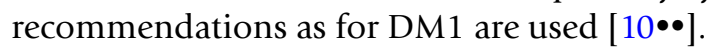


Disordered sleep due to obstructive sleep apnea, restless legs syndrome, REM-behavior disorder, and periodic limb movement syndrome have been frequently observed in DM2 patients; therefore, if theses disturbance are suspected a polysomnography should be performed [75, 76, 77•].

Endocrine system

The most frequent endocrine comorbidities observed in DM2 are insulin resistance/diabetes mellitus, hypothyroidism, and hypogonadism [2, 5]. Furthermore, dyslipidemia and altered calcium metabolism have been often observed in DM1 and might occur also in DM2 [78]. Annual laboratory screening tests including HbA1c, glucose, thyroid hormones and antibodies,

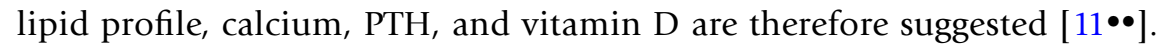
Further investigations as thyroid ultrasound, sexual hormones assessments, or glucose tolerance test might then be performed if abnormalities are detected. The treatment of the abovementioned endocrinological disorders does not differ from that of patients without myotonic dystrophy.

The involvement of the CNS has been well documented in DMs. Patients with DM2 show on neuropsychological tests mild frontal lobe impairments and avoidant personality traits, usually not impacting patients daily activities $[34 \bullet \bullet]$. Furthermore, affective disorders, especially depression, may be observed. Another prevalent and bothersome symptom is fatigue, probably of central origin, occurring with similar frequency in DM1 and DM2, often in combination with chronic pain and depression [79•]. It is therefore advisable to perform neuropsychological tests at diagnosis and to inquire patients about cognitive and psychiatric disorders during yearly neurological followup visits; if such symptoms emerge, repeating neuropsychological tests and

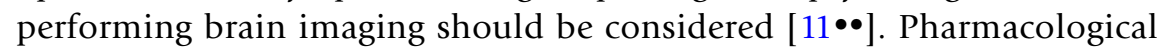
and psychological treatments should be adopted, if indicated, also to break the vicious circle of depression, chronic pain, reduced mobility, and fatigue. Interesting results were obtained in the OPTIMISTIC trial on patients with DM1 and prominent fatigue, where the use of cognitive behavioral therapy improved patients' activity and participation [80••].

Annual ophthalmological examinations allow the early detection and treatment of presenile cataract and other ocular abnormalities as epiretinal membrane $\left[81^{\bullet}\right.$ ]. Regular examinations should continue also after cataract surgery as cataract might recur $[11 \bullet \bullet]$. 
Several gastrointestinal signs and symptoms are described in patients with DM1 and DM2 including diarrhea/constipation, gastroesophageal reflux, abdominal pain, gallstones, and non-alcoholic fatty liver [82]. If patients present any of these complaints, a gastroenterological examination should be performed to exclude causes other than DM2 and then specific/symptomatic treatment can be initiated.

A higher incidence of tumors has been documented in DM1 in comparison to the general population (hazard ratio 1.81); it is unclear if this regard also DM2 patients, where tumors occur less frequently than in DM1 [66, 83]. In order to detect tumors early, patients must be advised to follow the current recommendations for cancer screening valid for the general population [11••].

Future therapies

\section{Conclusions}

At the moment, a long list of investigational products is being investigated for the treatment of the root causes of myotonic dystrophies (https://www.myotonic.org/ sites/default/files/pages/files/Myotonic-Dystrophy-Drug-Development-Pipelineas-of-March-8th-2021.pdf). Currently, all products in the clinical and preclinical

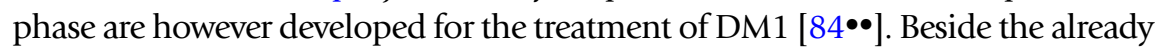
cited phase III study with metformin, the most advanced drug in the pipeline is tideglusib, a GSK3b inhibitor that showed promising results in the phase II trial on patients with congenital and childhood-onset DM1 [85••]. Given the specific mechanism of action on the CELF1/GSK3b pathway, mainly involved in DM1, this small molecule may be not suitable for the treatment of DM2 patients. An alternative mechanism of action of small molecules is the inhibition of the binding between toxic RNAs and MBNL1, relevant for both DMs [86•, 87•]. Erythromycin rescued mis-splicing events and improved myotonia in mice and will probably soon be tested in a phase II study on DM1 patients [86•, 87•]. Another very much studied therapeutic approach aims at post-transcriptional knockdown of toxic RNA using antisense oligonucleotides (ASOs) [88]. Many different ASOs are being tested in the preclinical stage in DM1 models [84 ••], their application for DM2 might be complicated by the concern that a further reduction of CNBP might be deleterious [88]. Lastly, big hopes and efforts are oriented to the use of the CRISPR/Cas9 genetic scissor as a tool to remove the (C)CUG DNA expansion; however, still many obstacles as efficient delivery and off-target gene editing need to be addressed before this therapeutic approach might be adopted in clinical trials [89••].

In conclusion, even if at the moment no treatments are available to drastically reverse the course of myotonic dystrophies, the use of high standards of care for the symptomatic management of symptoms and early detection of comorbidities should be pursued and has a positive impact on patient's quality of life. In order to achieve this, a holistic approach considering the complex multifaceted nature of DM should be chased with the help of a multidisciplinary team experienced in the treatment of DM. 
More efforts should be oriented to delineate the natural history of DM2 by designing longitudinal multicentric studies, as the clinical differences with DM1 need to be further investigated and translated into DM2 specific recommendations.

\section{Funding}

Open Access funding enabled and organized by Projekt DEAL.

\section{Compliance with Ethical Standards}

\section{Conflict of interest}

Federica Montagnese declares that she has no conflict of interest.

\section{Human and Animal Rights and Informed Consent}

This article does not contain any studies with human or animal subjects performed by any of the authors.

Open Access This article is licensed under a Creative Commons Attribution 4.0 International License, which permits use, sharing, adaptation, distribution and reproduction in any medium or format, as long as you give appropriate credit to the original author(s) and the source, provide a link to the Creative Commons licence, and indicate if changes were made. The images or other third party material in this article are included in the article's Creative Commons licence, unless indicated otherwise in a credit line to the material. If material is not included in the article's Creative Commons licence and your intended use is not permitted by statutory regulation or exceeds the permitted use, you will need to obtain permission directly from the copyright holder. To view a copy of this licence, visit http://creativecommons.org/licenses/by/4.0/.

\section{References and Recommended Reading}

Papers of particular interest, published recently, have

been highlighted as:

- Of importance

• Of major importance

1. Liquori CL, Ricker K, Moseley ML, et al. Myotonic dystrophy type 2 caused by a CCTG expansion in intron I of ZNF9. Science (80- ) 2001;293:864-7.

2. Day JW, Ricker K, Jacobsen JF, et al. Myotonic dystrophy type 2: molecular, diagnostic and clinical spectrum. Neurology. 2003;60:657-64.

3. Saito T, Amakusa Y, Kimura T, et al. Myotonic dystrophy type 2 in Japan: ancestral origin distinct from Caucasian families. Neurogenetics. 2008;9:61-3.

4. Logician EL, Ciafaloni E, Quinn LC, et al. Severity, type, and distribution of myotonic discharges are different in type 1 and type 2 myotonic dystrophy. Muscle Nerve. 2007;35:479-85.
5. Montagnese F, Mondello S, Wenninger S, et al. Assessing the influence of age and gender on the phenotype of myotonic dystrophy type 2. J Neurol 2017;264.

6. Hilbert JE, Ashizawa T, Day JW, et al. Diagnostic odyssey of patients with myotonic dystrophy. J Neurol. 2013;260:2497-504.

7. Tieleman AA, Jenks KM, Kalkman JS, et al. High disease impact of myotonic dystrophy type 2 on physical and mental functioning. J Neurol. 2011;258:1820-6.

8. Heatwole C, Bode R, Johnson N, et al. Patientreported impact of symptoms in myotonic dystrophy type 1 (PRISM-1). Neurology. 2012;79:348-57. 
9.• McNally EM, Mann DL, Pinto Y, et al. Clinical care recommendations for cardiologists treating adults with myotonic dystrophy. J Am Heart Assoc 2020;9:1-7.

Updated and complete recommendations for monitoring and treatment of cardiac involvement in patients with DM1, valid also for DM2

$10 . \bullet$ Boentert M, Cao M, Mass D, et al. Consensus-based care recommendations for pulmonologists treating adults with myotonic dystrophy type 1 .

Respiration 2020;99:360-8. Updated and complete recommendations for monitoring and treatment of respiratory involvement for patients with DM1, only partly applicable for DM2.

11.• Schoser B, Montagnese F, Bassez G, et al. Consensusbased care recommendations for adults with myotonic dystrophy type 2. Neurol Clin Pract 2019;9.

Expert's recommendations for optimized standards of care specific for patients with DM2.

12. Benhalevy D, Gupta SK, Danan $\mathrm{CH}$, et al. The Human CCHC-type zinc finger nucleic acid-binding protein binds g-rich elements in target mRNA coding sequences and promotes translation. Cell Rep 2017;18:2979-90.

13. Raheem O, Olufemi SE, Bachinski LL, et al. Mutant (CCTG)n expansion causes abnormal expression of zinc finger protein 9 (ZNF9) in myotonic dystrophy type 2. Am J Pathol. 2010;177:3025-36.

14. Sansone VA, Brigonzi E, Schoser B, et al. Haploinsuffciency for $\mathrm{Znf9}$ in $\mathrm{Znf9+/-} \mathrm{mice} \mathrm{is} \mathrm{associated} \mathrm{with}$ multiorgan abnormalities resembling myotonic dystrophy. Neurology. 2014;365:8-17.

15. Cox DC, Guan X, Xia Z, Cooper TA. Increased nuclear but not cytoplasmic activities of CELF1 protein leads to muscle wasting. Hum Mol Genet 2020;29:1729-44.

Inetersting study clarifying the role of CELF1 in muscle wasting in DM1.

16. Wang ET, Ward AJ, Cherone JM, et al. Antagonistic regulation of mRNA expression and splicing by CELF and MBNL proteins. Genome Res. 2015;25:858-71.

17. Dong W, Chen X, Wang M, et al. Mir-206 partially rescues myogenesis deficiency by inhibiting CUGBP1 accumulation in the cell models of myotonic dystrophy. Neurol Res 2019;41:9-18.

Pathogenic insights into function of CUGBP1 in DM.

18. Cardani R, Bugiardini E, Renna L V., et al. Overexpression of CUGBP1 in skeletal muscle from adult classic myotonic dystrophy type 1 but not from myotonic dystrophy type 2. PLoS One 2013;8.

$19 . \bullet$ Sellier C, Cerro-Herreros E, Blatter M, et al. RbFOX1/ MBNL1 competition for CCUG RNA repeats binding contributes to myotonic dystrophy type 1/type 2 differences. Nat Commun 2018;9.

In this study the author demonstrate that RbFox1 plays a central role in DM2 pathogenesis and phenotypic differences with DM1.

20. Zu T, Cleary JD, Liu Y, et al. RAN Translation regulated by muscleblind proteins in myotonic dystrophy type 2. Neuron. 2017;95:1292-1305.e5.
21.• Castelli LM, Huang W-P, Lin Y-H, et al. Mechanisms of repeat-associated non-AUG translation in neurological microsatellite expansion disorders. Biochem Soc Trans 2021.

Review on the mechanisms involved in RAN translation in myotonic dystrophies and other diseases.

22. SK T, L N, K T, et al. OUP accepted manuscript. Hum Mol Genet 2021.

23. Choi J, Personius KE, DiFranco M, et al. Muscleblindlike 1 and muscleblind-like 3 depletion synergistically enhances myotonia by altering Clc-1 RNA translation. EBioMedicine. 2015;2:1034-47.

24. Metzger S, Dupont C, Voss AA, Rich MM. Central role of subthreshold currents in myotonia. Ann Neurol 2020;87:175-83.

In this study the authors clarify the pathophysiology underlying myotonia and in particular the role of subtreshold currents. 25. Wheeler TM, Lueck JD, Swanson MS, et al. Correction of ClC-1 splicing eliminates chloride channelopathy and myotonia in mouse models of myotonic dystrophy. J Clin Invest. 2007;117:3952-7.

26. Tan SV, Z'Graggen WJ, Hanna MG, Bostock H. In vivo assessment of muscle membrane properties in the sodium channel myotonias. Muscle Nerve. 2018;57:586-94.

27. Hawash AA, Voss AA, Rich MM. Inhibiting persistent inward sodium currents prevents myotonia. Ann Neurol. 2017;82:385-95.

28.• Dupont C, Novak K, Denman K, et al. TRPV4 Antagonism prevents mechanically induced myotonia. Ann Neurol 2020;88:297-308.

Interesting study demonstrating that the mechanisms of percussion myotonia differ from those of action myotonia.

29. Portaro S, Russo M, Naro A, et al. Advances in assessing myotonia: can sensor-engineered glove have a role? J Neurol Sci. 2017;375:3-7.

30. Statland JM, Bundy BN, Wang Y, et al. A quantitative measure of handgrip myotonia in non-dystrophic myotonia. Muscle Nerve. 2012;46:482-9.

31. Kronlage C, Grimm A, Romano A, et al. Muscle ultrasound shear wave elastography as a non-invasive biomarker in myotonia. Diagnostics 2021;11:163.

Study highlighting the potential use of ultrasound as a diagnostic and outcome measure for myotonia.

32. Abraham A, Breiner A, Barnett C, et al. Quantitative sonographic assessment of myotonia. Muscle and Nerve 2018;57:146-9.

Study highlighting the potential use of ultrasound as a diagnostic and outcome measure for myotonia.

33. Heatwole C, Johnson N, Bode R, et al. PatientReported Impact of Symptoms in Myotonic Dystrophy Type 2 (PRISM-2). Neurology. 2015;85:2136-46.

$34 . \bullet$ Meola G. Myotonic dystrophy type 2: the 2020 update. ACTA Myol 2020;XXXIX:222-34.

Complete, updated review on DM2.

35. Cardani R, Giagnacovo M, Botta A, et al. Co-segregation of DM2 with a recessive CLCN1 mutation in juvenile onset of myotonic dystrophy type 2 . J Neurol. 2012;259:2090-9. 
36. Bugiardini E, Rivolta I, Binda A, et al. SCN4A mutation as modifying factor of Myotonic Dystrophy Type 2 phenotype. Neuromuscul Disord. 2015;25:301-7.

37. Passeri E, Sansone VA, Verdelli C, et al. Asymptomatic myotonia congenita unmasked by severe hypothyroidism. Neuromuscul Disord. 2014;24:365-7.

38. Desaphy JF, Carbonara R, Costanza T, Conte CD. Preclinical evaluation of marketed sodium channel blockers in a rat model of myotonia discloses promising antimyotonic drugs. Exp Neurol. 2014;255:96-102.

39.• Desaphy JF, Farinato A, Altamura C, et al. Safinamide's potential in treating nondystrophic myotonias: Inhibition of skeletal muscle voltage-gated sodium channels and skeletal muscle hyperexcitability in vitro and in vivo. Exp Neurol 2020;328

In this study the anti-myotonic effect of safinamide is proven and its potential clinical application discussed.

40. Montagnese F, Stahl K, Wenninger S, Schoser B. A role for cannabinoids in the treatment of myotonia? Report of compassionate use in a small cohort of patients. J Neurol 2020;267.

In this case series a small number of patients with myotonic syndrome have experienced improvement of myalgia and myotonia with a combination of THC and CBD.

41.• Montagnese F, White M, Klein A, et al. Cannabis use in myotonic dystrophy patients in Germany and USA: a pilot survey. J Neurol 2019;266.

This survey highlights the need for furthe research on the therapeutic potential of cannabinoids in the treatment of DM. 42.• Beauchesne W, Savard C, Côté-Hamel M, et al. Characterization of cannabis use by patients with myotonic dystrophy type 1: A pilot study. Neuromuscul Disord 2021;31:226-31.

This study highlights again the widespread use of cannabinoids in the DM1 population and the need for further research.

43. Logigian EL, Martens WB, Moxley RT, et al. Mexiletine is an effective antimyotonia treatment in myotonic dystrophy type 1. Neurology. 2010;74:1441-8.

$44 . \bullet$ Heatwole C, Luebbe E, Rosero S, et al. Mexiletine in myotonic dystrophy type 1: a randomized, doubleblind, placebo-controlled trial. vol. 96. NLM (Medline); 2021.

RCT proving safety and efficacy of Mexiletine in DM1.

$45 . \bullet$ Vio R, Zorzi A, Bello L, et al. Evaluation of mexiletine effect on conduction delay and bradyarrhythmic complications in patients with myotonic dystrophy type 1 over long-term follow-up. Hear Rhythm 2020;17:1944-50.

Study proving the favorable safety profile in regards to cardiac complications of Mexiletine in DM1.

46. Montagnese F, Rastelli E, Khizanishvili N, et al. Validation of Motor Outcome Measures in Myotonic Dystrophy Type 2. Front Neurol 2020;11.

Study investigating the applicability of outcome measures in DM2 patients

47.• Puntillo F, Giglio M, Paladini A, et al. Pathophysiology of musculoskeletal pain: a narrative review. Ther Adv Musculoskelet Dis 2021;13.
Interesting review on the mechanisms of muskuloskeletal pain with implications for DM2 research

48. George A, Schneider-Gold C, Zier S, et al. Musculoskeletal pain in patients with myotonic dystrophy type 2. Arch Neurol 2004;61:1938-42

49. Suokas KI, Haanpää M, Kautiainen H, et al. Pain in patients with myotonic dystrophy type 2 : a postal survey in Finland. Muscle Nerve. 2012;45:70-4.

50. van Vliet J, Tieleman AA, Verrips A, et al. Qualitative and quantitative aspects of pain in patients with myotonic dystrophy type 2. vol. 19. Elsevier Inc.; 2018.

51. Moshourab R, Palada V, Grunwald S, et al. A Molecular Signature of Myalgia in Myotonic Dystrophy 2. EBIOM. 2016;7:205-11.

52. Arendt-Nielsen L, Fernández-de-las-Peñas C, GravenNielsen T. Basic aspects of musculoskeletal pain: From acute to chronic pain. J Man Manip Ther. 2011;19:186-93.

53. Schneider-Gold C, Bellenberg B, Prehn C, et al. Cortical and subcortical grey and white matter atrophy in myotonic dystrophies type 1 and 2 is associated with cognitive impairment, depression and daytime sleepiness. PLoS One 2015;10.

54. Tieleman AA, Knoop H, Van De Logt AE, et al. Poor sleep quality and fatigue but no excessive daytime sleepiness in myotonic dystrophy type 2. J Neurol Neurosurg Psychiatry. 2010;81:963-7.

55. Stockings E, Campbell G, Hall WD, et al. Cannabis and cannabinoids for the treatment of people with chronic noncancer pain conditions: a systematic review and meta-analysis of controlled and observational studies. Pain. 2018;159:1932-54.

56.• El-Tallawy SN, Nalamasu R, Salem GI, et al. Management of musculoskeletal pain: an update with emphasis on chronic musculoskeletal pain. Pain Ther 2021:1-29.

Interesting review on the best pharmacological and nonpharmacological treatment of chronic muscle pain.

57. Tieleman AA, Knuijt S, van Vliet J, et al. Dysphagia is present but mild in myotonic dystrophy type 2 . Neuromuscul Disord. 2009;19:196-8.

58. Schneider-Gold C, Beck M, Wessig C, et al. Creatine monohydrate in DM2/PROMM: a doubleblind placebo-controlled clinical study. Neurology. 2003;60:500-2.

59. Roussel MP, Morin M, Gagnon C, Duchesne E. What is known about the effects of exercise or training to reduce skeletal muscle impairments of patients with myotonic dystrophy type 1 ? A scoping review. BMC Musculoskelet Disord 2019;20.

Review summarizing the results of various exercise training programs in patients with DM1.

60. Kontou E, Papadopoulos C, Papadimas G, et al. Effect of exercise training on functional capacity and body composition in myotonic dystrophy type 2 patients. Muscle Nerve 2021;63:477-83.

Study highlighting the beneficial effect of exercise in patients with DM2. 
61.• Bassez G, Audureau E, Hogrel JY, et al. Improved mobility with metformin in patients with myotonic dystrophy type 1 : a randomized controlled trial. Brain 2018;141:2855-65.

RCT demonstrating the efficacy of metformin in imporoving mobility of patients with DM1

62. Laustriat D, Gide J, Barrault $\mathrm{L}$, et al. In vitro and in vivo modulation of alternative splicing by the biguanide metformin. Mol Ther - Nucleic Acids 2015;4:e262.

63. Nieuwenhuis S, Okkersen K, Widomska J, et al. Insulin signaling as a key moderator in myotonic dystrophy type 1 . Front Neurol 2019;10:1-17.

Insights into the multifaceted pathogenic pathways involving insulin signaling with implications for myotonic dystrophy pathogensis and therapeutic approaches.

64. García-Puga M, Saenz-Antoñanzas A, Fernández-Torrón $\mathrm{R}$, et al. Myotonic Dystrophy type 1 cells display impaired metabolism and mitochondrial dysfunction that are reversed by metformin. Aging (Albany NY) 2020;12:6260-75.

Study investigating the mechanism of action of metformin in DM1.

65. Mathieu J, Allard P, Potvin L, et al. A 10-year study of mortality in a cohort of patients with myotonic dystrophy. Neurology 1999;52:1658-62

66. Gadalla SM, Pfeiffer RM, Kristinsson SY, et al. Quantifying cancer absolute risk and cancer mortality in the presence of competing events after a myotonic dystrophy diagnosis. PLoS One 2013;8.

67. Ashizawa T, Gagnon C, Groh WJ, et al. Consensusbased care recommendations for adults with myotonic dystrophy type 1 . Neurol Clin Pract. 2018;8:507-20.

68.• Wahbi K, Furling D. Cardiovascular manifestations of myotonic dystrophy. Trends Cardiovasc Med 2020;30:232-8.

Review with a detailed presentation of the possible cardiac complications in patients with DM.

69. Garibaldi M, Lauletta A, Bucci E, et al. Gender effect on cardiac involvement in myotonic dystrophy type 1. Eur J Neurol 2020.

Study highlighting the higher risk of cardiac complications in males than females with DM1 with possible implications for patients' monitoring.

70. Joosten IBT, van Lohuizen R, den Uijl DW, et al. Electrocardiographic predictors of infrahissian conduction disturbances in myotonic dystrophy type 1 . EP Eur 2020:1-7.

Study highlighting the hints that might reveal a infrahissian conduction disturbance and therefore require invasive electrophysiological investigations

71. Vujnic M, Peric S, Popovic S, et al. Metabolic syndrome in patients with myotonic dystrophy type 1 . Muscle Nerve. 2015;52:273-7.

72.• Hawkins AM, Hawkins CL, Abdul Razak K, et al. Respiratory dysfunction in myotonic dystrophy type 1: a systematic review. Neuromuscul Disord 2019;29:198-212.
Review of studies on respiratory function assessed in DM1 with implications for monitoring and treatment of patients. 73. Sansone VA, Gagnon C, Atalaia A, et al. 207th ENMC Workshop on chronic respiratory insufficiency in myotonic dystrophies: management and implications for research, 27-29 June 2014, Naarden. The Netherlands Neuromuscul Disord. 2015;25:432-42.

74.• Romigi A, Maestri M, Nicoletta C, et al. Sleep complaints, sleep and breathing disorders in myotonic dystrophy type 2. Curr Neurol Neurosci Rep 2019;19.

Study highlighting the most common findings of disordered sleep in patients with DM2.

75. Tieleman AA, Knoop H, Van De Logt AE, et al. Poor sleep quality and fatigue but no excessive daytime sleepiness in myotonic dystrophy type 2. J Neurol Neurosurg Psychiatry. 2010;81:963-7.

76. Romigi A, Franco V, Placidi F, et al. Comparative sleep disturbances in myotonic dystrophy types 1 and 2. Curr Neurol Neurosci Rep 2018;18.

77. $\quad$ Subramony SH, Wymer JP, Pinto BS, Wang ET. Sleep disorders in myotonic dystrophies. Muscle and Nerve 2020;62:309-20.

Review on the most common sleep disturbances observed in DM.

78. Ørngreen MC, Arlien-Søborg P, Duno M, et al. Endocrine function in 97 patients with myotonic dystrophy type 1. J Neurol 2012;259:912-20.

79.• Gliem C, Minnerop M, Roeske S, et al. Tracking the brain in myotonic dystrophies: a 5-year longitudinal follow-up study. PLoS One 2019;14.

One of the few longitudinal study on brain involvement in DM highlighting a very slow progression.

80.• Okkersen K, Jimenez-Moreno C, Wenninger S, et al. Cognitive behavioural therapy with optional graded exercise therapy in patients with severe fatigue with myotonic dystrophy type 1: a multicentre, single-blind, randomised trial. Lancet Neurol 2018;17:671-80. Relevant study hihlighting the benefit of CBT in DM1 for improved mobility and fatigue, also highlighting good patient's compliance.

81. Abed E, D'Amico G, Rossi S, et al. Spectral domain optical coherence tomography findings in myotonic dystrophy. Neuromuscul Disord 2020;30:144-50.

Recent study on common ocular findings in DM.

82. Tieleman AA, van Vliet J, Jansen JBMJ, et al. Gastrointestinal involvement is frequent in Myotonic Dystrophy type 2. Neuromuscul Disord 2008;18:646-9

83. Alsaggaf R, St. George DMM, Zhan M, et al. Cancer risk in myotonic dystrophy type $\mathrm{i}$ : evidence of a role for disease severity. JNCI Cancer Spectr 2018;2:1-7.

84.• Pascual-Gilabert M, López-Castel A, Artero R. Myotonic dystrophy type 1 drug development: a pipeline toward the market. Drug Discov Today 2021.

Clear and updated review on the future therapeutic options for DM.

85.• Horrigan J, Gomes TB, Snape M, et al. A phase 2 study of AMO-02 (Tideglusib) in congenital and childhood-onset myotonic dystrophy type 1 (DM1). Pediatr Neurol 2020;112:84-93. 
Study proving the efficacy of tideglusib for CMD and childhood DM1. A phase III trial will follow.

86. Jenquin JR, Yang H, Huigens RW, et al. Combination treatment of erythromycin and furamidine provides additive and synergistic rescue of mis-splicing in myotonic dystrophy type 1 models. ACS Pharmacol Transl Sci 2019;2:247-63.

In vivo study highlighting potential clinical benefit of erythromycin in DM1

87.• Nakamori M, Taylor K, Mochizuki H, et al. Oral administration of erythromycin decreases RNA toxicity in myotonic dystrophy. Ann Clin Transl Neurol 2016;3:42-54

In vivo study highlighting potential clinical benefit of erythromycin in DM1

88. Thornton CA, Wang E, Carrell EM. Myotonic dystrophyapproach to therapy. Curr Opin Genet Dev. 2017;44:135-40.
89.• Marsh S, Hanson B, Wood MJA, et al. Application of CRISPR-Cas9-mediated genome editing for the treatment of myotonic dystrophy type 1 . Mol Ther 2020;28:2527-39.

Nice review on the current challenges of CRIPR/Cas technology in DM.

\section{Publisher's Note}

Springer Nature remains neutral with regard to jurisdictional claims in published maps and institutional affiliations. 